\section{Identification and analysis of putative promoter motifs in bovine herpes virus}

\author{
Mahantesh Mallikrjun Kurjogi', Rajeshwari Danappa Sanakal2 \& Basappa Basaveneppa \\ Kaliwal ${ }^{1 *}$
}

IPG. Dept of Studies in Microbiology and Biotechnology Karnatak University, Dharwad-58003, Karnataka, India; 2Department of Zoology, Karnatak Science College, Dharwad-58003, Karnataka, India; Basappa Basaveneppa Kaliwal - Email: b_kaliwal@yahoo.com; *Corresponding author

Received August 11, 2012; Revised September 28, 2012; A ccepted November 03, 2012; Published N ovember 23,2012

\begin{abstract}
A bstract:
The purpose of this study is to identify and analyse the putative promoter motifs in the bovine herpes virus which causes several diseases in cattle worldwide induding bovine mastitis with large economic impact on dairy industry. Bovine mastitis caused due to virus is often neglected as bacterial infections are held mainly responsible for the disease. Therefore, in this in silico investigation with all the existing experimental data a total of 147 promoter were identified al ong with their sequences from three genome viz bovine herpes virus 1 (BHV), bovine herpes virus 4 and bovine herpes virus 5 , out of which 39 promoters were from bovine herpes virus 4 (BHV 4), 95 from BHV1 and 13 from BHV5 and it was observed that BHV1 and BHV5 have a close evolutionary history. However, they belong to the same subfamily and size of the genome and GC\% of BHV1 and BHV5 was almost equal and very high compare to that of BHV4. This analysis may help in designing the live attenuated vaccine against BHV causing bovine mastitis that reduces the incidence of bovine mastitis. Identification of promoters may also help in designing of expression vectors which help in better understanding of the regulation of gene expression. In the era of large genomics and proteomics prediction of promoters in the whole genome is crucial for the advancement of drug discovery and genetherapy.
\end{abstract}

Keyw ords: Bovine herpes virus, bovine mastitis, promoters, transcription factors, genome.

\section{Background:}

Bovine mastitis is frequently occurring disease affecting dairy cattle with large economic losses in dairy industries. Though bacteria are the main causative agents of bovine mastitis, still there are 20 - 35\% cases of bovine mastitis which are bacteriologically negative for the milk samples [1, 2] perhaps there may be pathogens other than bacteria causing disease, reports suggests that bovine herpes virus 4 is isolated from the milk of cows with mastitis [3]. Bovine herpes virus 4 is also isolated from the milk of staphylococcus mastitis. Reports suggests that there is a positive association between the bovine herpes virus 4 seropositivity of cows and the incidence of bovine mastitis caused by S. aureus [4] and S.aureus is found to be the main causative agent of bovine mastitis [5].

Bovine herpes virus 4 is a member of the family herpesviridae, subfamily gamma herpesvirinae belonging to the order herpesvirales and genes rhadinovirus. BHV 4 has an enveloped icosahedral nucleocapsid, with a diameter of $100 \mathrm{~nm}$, while the overall diameter of the virus particle is approximately $150 \mathrm{~nm}$. The present study was also extended for the analysis of the genome of BHV1 and BHV5 which belongs to order herpesvirales family herpesviridae, subfamily al phaherpesvirinae, genus varicellovirus. BHV1 and $\mathrm{BHV} 5$ are held responsible for causing the most important emerging diseases of dairy cattle in many countries of the world. The disease caused by BHV in dairy cattle is characterized by the signs of respiratory disorder, general illness, abortion and reduced quality and quantity of milk yield. Virus has a wide host range which is known to infect different ruminant species like cattle, sheep and goats [6, 7]. BHV needs dividing cells for effective virus replication as there is increase in viral DNA replication and protein expression at the $S$ phase of the cell cycle. 
The regulation of gene expression can be controlled at transcription level by a promoter region that contains a specific DNA sequence from which transcription begins and proceeds through the coding sequences and ends at the terminator site. The sequence of the promoters affects the transcription initiation event and also influences the rate at which RNA polymerase clears the promoter region to begin elongation in a typical transcription process. Therefore, identification of promoter sequences in a whole genome is crucial for regulation of gene expression in BHV. However, a limited number of data is available on the BHV. Hence, the present study was carried out through a computational method for the identification and analysis of putative promoter motifs in BHV.

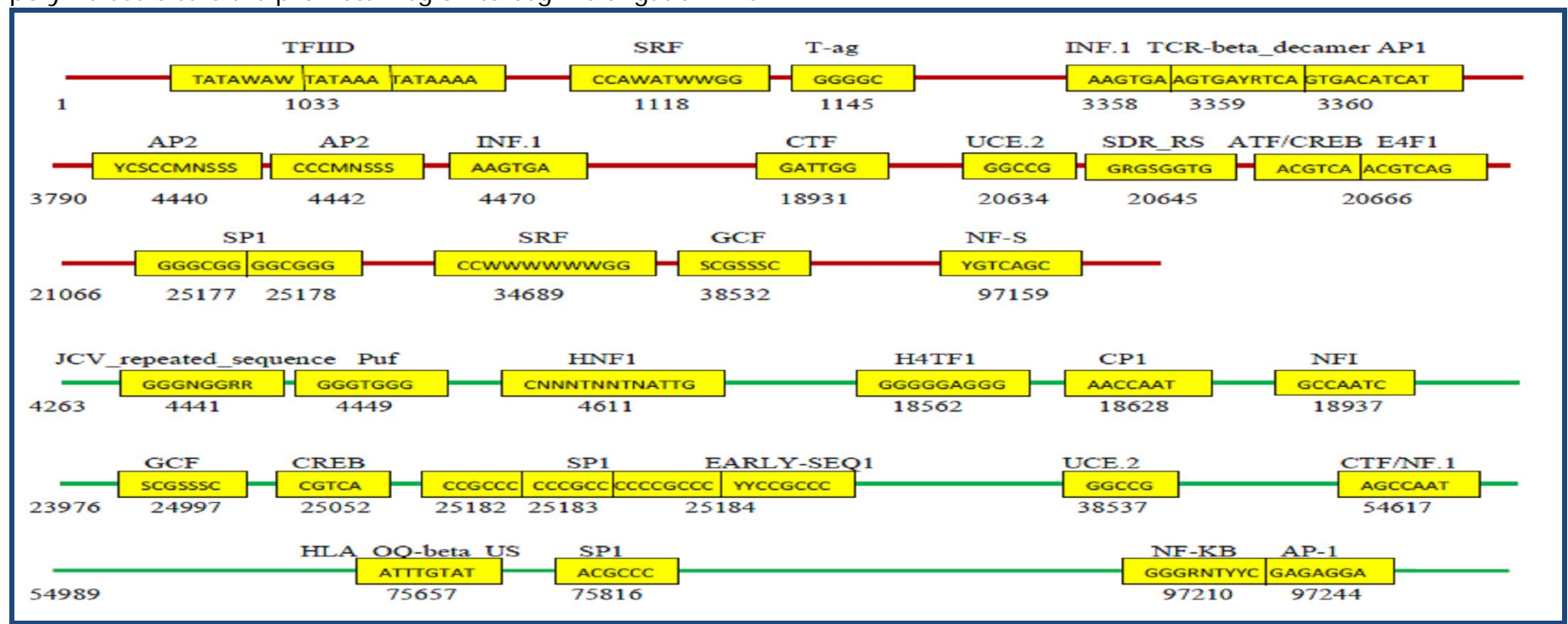

Figure 1: The promoters of Bovine Herpes virus 4.

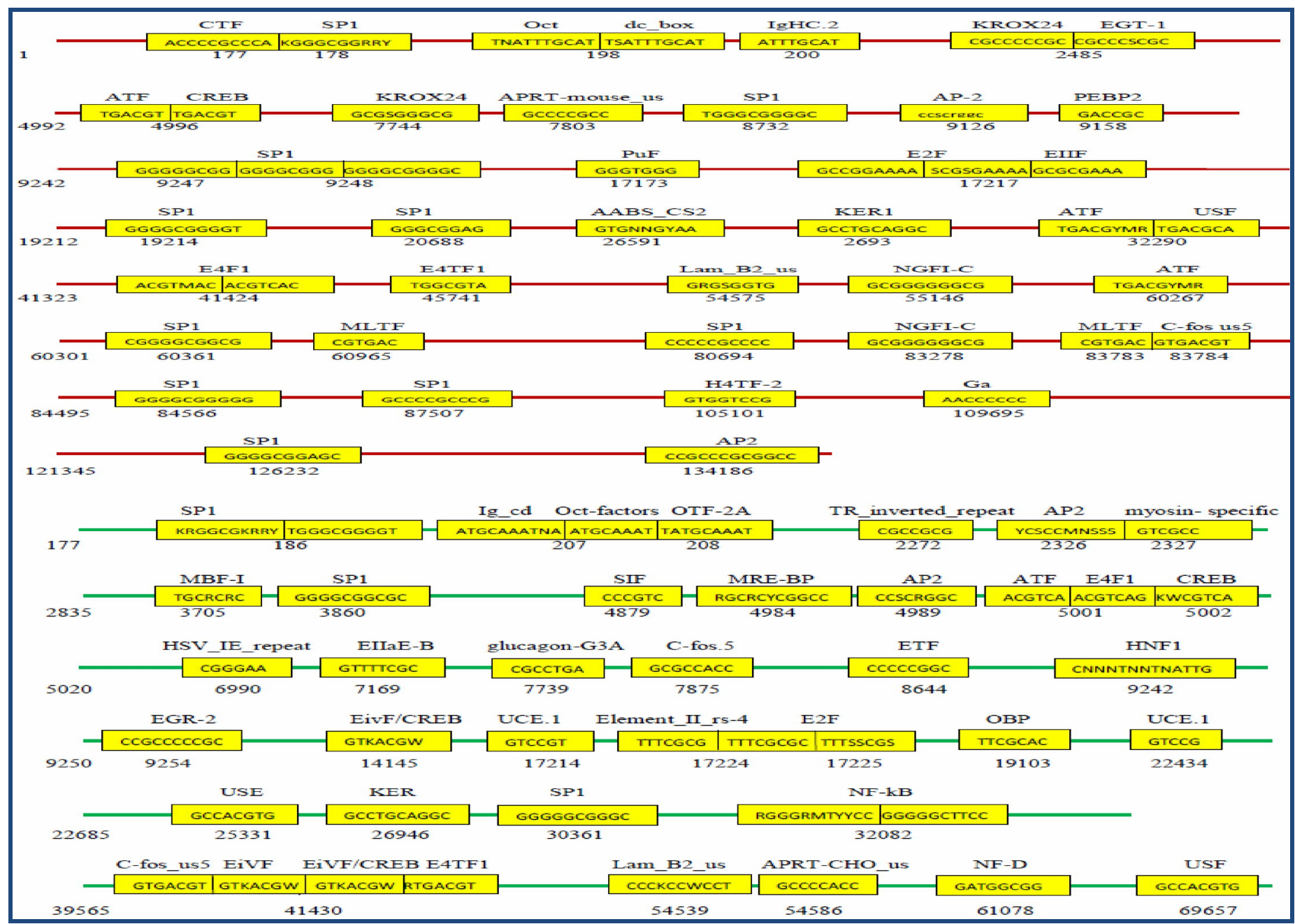

Figure 2: The promoters of BovineH erpes virus 1. 


\section{Methodology}

\section{Retrieval of genome sequence}

The complete genome sequences of BHV were retrived from biological database such as National centre for Biotechnology Information

( $\mathrm{NCBI})$ cited

http:/ / www.ncbi.nlm.nih.gov/ genomes/ viruses.html.

\section{Analysis of BHV genomes}

The size of the genome of bovine herpes virus (BHV) was analysed in the FASTA format the $\mathrm{G}+\mathrm{C} \%$ was compared and total number of genes werealso noted.

\section{Transcription promoter site}

The putative promoter in the genome of BHV was identified by using the promoter scan programme at http:/ / wwwbimas.cit.nih.gov/molbio/ proscan/. The complete genome BHV1, BHV4 and BHV5 was used for the analysis of promoters in all three genomes. The program comprises three databases such as TF databases, promoter databases and non promoter set constructed from protein and RNA gene sequences. In this study we have provided a brief description of putative promoters of Bovine herpes virus. However, there are numerous methodologies available in the public domain for the analysis of promoters but still further validation is needed for a researcher before picking up the problem for investigation in the molecular biology laboratory.

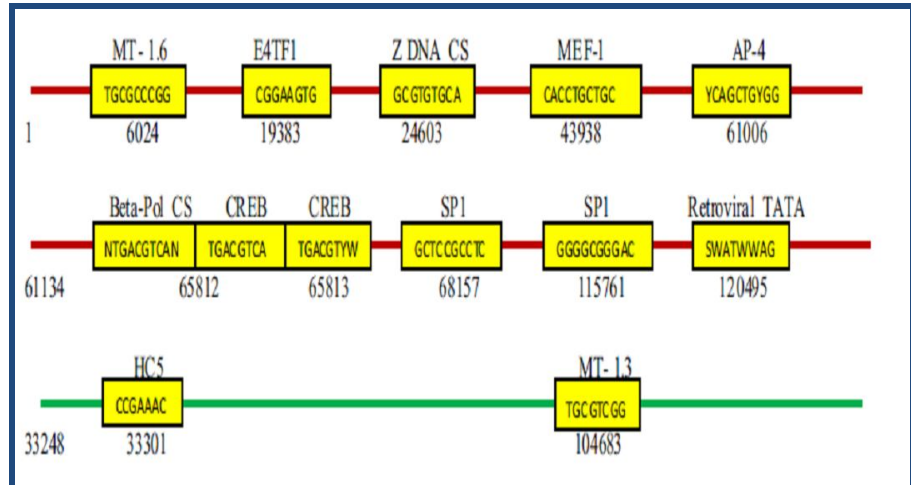

Figure 3: The promoters of Bovine Herpes virus 5. - The red line indicates the positive strand; - The green line indicates the negative strand; $\square$ The yellow box indicates the promoter sequence and the number below the box indicates the specific location of the promoter. The letters above the box indicates the promoter designation.

\section{Results and Discussion:}

In the present study complete genome sequence of $\mathrm{BHV} 4$, BHV1 and BHV5 was retrieved from the accession on NC 002665.1, NC 001847.1 and NC 005261.2 and the size of genome was found to be $108.87 \mathrm{~kb}, 135.3 \mathrm{~kb}$ and $137.87 \mathrm{~kb}$ respectively the highest GC \% was found in $74.8 \%$ in BHV 5 followed by BHV1 $72.4 \%$ and BHV4 41.4\% the highest number of genes were found in BHV 479 followed by BHV1 and BHV5 70 genes each. Similarly reports suggest that the genome size of Kamati River and Tick borne encephalitis virus was $11 \mathrm{~kb}$, Tamana bat virus was 10,053 bases and GC\% of Tamana bat virus and Louping ill virus was 38.43 and 54.85 respectively [8]. DNA with high GC-content is more stable than DNA with low GC-content. GC base pairs are more stable than AU base pairs, due to the fact that $\mathrm{GC}$ bonds have 3 hydrogen bonds and $A U$ only has 2 hydrogen bonds, which makes high-GC-content RNA structures more tolerant to high temperatures. More recently, the first large-scale systematic gene-centric association analysis demonstrated the correlation between GC content and temperature for certain genomic regions while not for others [9]. Further, the identification of putative promoter in the genome of BHV1, BHV4 and BHV5 was also carried out. A total of 147 types of promoters and their sequences were identified in the genomes (Fig 1, 2 and 3) most of the promoters found in all 3 genomes are similar and it is confirmed with the existing data.

A very limited data is available on the identification and charecterization of promoter in the virus genome. Transcription factor IID (TFIID) is one of the general transcription factors that make up the RNA polymerase II preinitiation complex [10] TFIID binds to TATA box in the core promoter of the gene It regulates the activities of more than 70 polypeptides required for the initiation of transcription by RNA polymerase II and also acts as channel for regulatory signals. SRF is a serum response element - binding transcription factor [11] that regulates the activity of many immediate early genes viz C-fos and thereby participates in cell cycle regulation, apoptosis, cell growth and cell differentiation. SV40 is a double stranded DNA virus which causes tumors at multiple sites to wide range of vertebrates. T- Ag is a protein of proto- oncogene present in the SV 40 and is involved in viral genome replication and regulation of host cell cycle[12].

AP1 (activating protein1) is a collective term referring to determine transcription factor composed of Jun, Fos or ATF subunit that bind to a common DNA site. Different AP1 factors may regulate different target genes and thus execute distinct biological functions [13]. NF1 gene promoter harbours a hypomethylated CpG island. Hence, methylation changes may be involved in the development of different types of neurofibromas and malignant transformation [14].

The human neurotropic papovirus JCV contains sequences within the two 98 -bp tandum repeat which play a key role in glial - specific transcription of the early and late stage of viral promoter sites [15]. TCR $\mathrm{V}$ beta promoter contains a highly conserved decamer homologous to CAMP response element (CRE). It has been shown that TCR beta - chain expression immediately activated CAMP. Such induction is likely to be mediated through $\mathrm{V}$ beta-CRE sequence because the inclusion of $\mathrm{V}$ - beta - CRE in a vector with minimum promoter (PB1 CAT2) conferred the CAMP inducibility of CAT activity [16].

The promoter of the early growth response gene (Egr-1) has been described to be activated by ionizing radiation it has been reported that a novel regulatory element in the human Egr-1 promoter is similar to a NF kappa-B binding site [17]. HNF-1 beta forms a homodimer or a hetrodimer with HNF1 alpha and regulates various target genes. HNF1 beta mutations are rare and no functional analysis has been performed in conjuction with HNF1 alpha. HNF1 beta controls liver specific and bile acid related genes as it is expressed in the liver and biliary system [18].

\section{Conclusion:}

In the present study attempts were made to find the promoter sites and regulation of genes and their function in the genomes 
with the available data by computational methods. A total of 147 promoters were identified from three bovine herpes virus genome out of which 39 promoters were from bovine herpes virus 4 (BHV 4), 95 from BHV1 and 13 from BHV5. The results of the present study may help in designing of the live attenuated vaccine by site directed mutagenesis in the promoter region which could be a permanent solution for the problem bovine mastitis due to Bovine herpes virus. This present study of promoters might also help in designing of expression vectors which helps in better understanding of regulation of gene expression. In the era of large genomics and proteomics prediction of promoters in the whole genome is crucial for drug discovery and gene therapy.

\section{Acknowledgement:}

The authors are grateful to Department of biotechnology, Ministry of Science and Technology, Government of India, New Delhi, for funding the Bioinformatics Infrastructure Facility Project, Interdisciplinary Program for Life Science Project and P. G Departments of Microbiology and Biotechnology Karnatak University, Dharwad for providing the facilities.

\section{References:}

[1] Miltenburg JD et al. Vet Rec. 1996 139: 204 [PMID: 8883335]

[2] Barkema HW et al. J D airySci. 1998 81: 411 [PMID: 9710760]
[3] Wellenberg G] et al. V et Rec. 2000 147: 222 [PMID: 10994925]

[4] Zadoks RN et al. J Dairy Sci. 2001 84: 2649 [PMID: 11814021]

[5] Kurjogi MM \& Kaliwal BB, Advances in Applied Science Research. 2011 2: 229

[6] VanOpdenbosch E et al. Vet Res Communications. 1988 12: 347

[7] Mahmoud MA \& A hmed G lobal SA, V eterinaria. 2009 3: 472

[8] Somvanshi $P$ et al. Bioinformation. 2008 3: 162 [PMID: 19238240]

[9] Zheng H \& Wu H, BM C Bioinformatics. 2010 11: S7 [PMID: 21172057]

[10] Lewin Benjamin, Genes VIII. 2004 pp. 636-637. ISBN 0-13144946-X

[11] N orman C et al. Cell. 1988 55: 989 [PMID: 3203386]

[12] Ali SH \& DeCaprio JA, Semin Cancer Biol. 2001 11: 15 [PMID: 11243895]

[13] Hess J et al. J Cell Sci. 2004 117: 5965 [PMID: 15564374]

[14] Horan MP et al. H um Genet. 2000 107: 33 [PMID: 10982032]

[15] Hiroomi T et al. Virology. 2004 180: 327

[16] LeeMR et al. J Immunol. 1992 148: 1906 [PMID: 1531847]

[17] Aicher WK et al. Rheumatol Int. 1999 18: 207 [PMID: 10399797]

[18] Kitanaka S et al. J Clin Endocrinol M etab. 2004 89: 1369 [PMID: 15001636]

Edited by $P$ Kangueane

Citation: K urjogi et al. Bioinformation 8(23): 1167-1170 (2012) License statement: This is an open-access article, which permits unrestricted use, distribution, and reproduction in any medium, for non-commercial purposes, provided the original author and source arecredited 